\title{
Resonant Anderson localization in segmented wires
}

\author{
Cristian Estarellas ${ }^{1}$ and Llorenç Serra ${ }^{1,2, *}$ \\ ${ }^{1}$ Institut de Física Interdisciplinària i de Sistemes Complexos, IFISC (CSIC-UIB), E-07122 Palma de Mallorca, Spain \\ ${ }^{2}$ Departament de Física, Universitat de les Illes Balears, E-07122 Palma de Mallorca, Spain
}

(Received 4 December 2015; published 4 March 2016)

\begin{abstract}
We discuss a model of random segmented wire, with linear segments of two-dimensional wires joined by circular bends. The joining vertices act as scatterers on the propagating electron waves. The model leads to resonant Anderson localization when all segments are of similar length. The resonant behavior is present with one and also with several propagating modes. The probability distributions evolve from diffusive to localized regimes when increasing the number of segments in a similar way for long and short localization lengths. As a function of the energy, a finite segmented wire typically evolves from localized to diffusive to ballistic behavior in each conductance plateau.
\end{abstract}

DOI: 10.1103/PhysRevE.93.032105

\section{INTRODUCTION}

The electrical transport properties of bent semiconductor nanowires have attracted much interest over the years [1-6]. In particular, phenomena such as the formation of localized states and the scattering behavior of circular bends were considered. At low energies it was proved that a circular bend can be understood as an attractive square well supporting bound states. Recently, we extended a similar analysis to closed polygons made of two-dimensional (2D) nanowires, finding characteristic sequences of eigenstates [7]. The optical absorption of polygonal nanorings was also recently considered in Ref. [8].

Motivated by the above studies, we present in this work a model of a segmented wire, made with a large collection of straight segments joined with circular bends (Fig. 1). We address the localization properties in the resulting nanowire when the vertices (bends) and segment lengths vary randomly. It is well known that the quantum interference of scattered waves in the presence of disorder leads to the phenomenon of Anderson localization [9]. Reviews of this persistent topic with extensive literature can be found, e.g., Refs. [10-15]. We address in this work the localization phenomenology of the segmented wire model. The limit of phase-coherent transport is assumed, with the bends acting as rigid and elastic scatterers, while any dynamic phase randomization such as that due to phonons is neglected.

Anderson localization in disordered 1D systems has been extensively investigated. In particular, the model of successive 1D barriers (or wells) of fixed thickness $\ell$ and random heights is known to lead to resonant localization whenever the accumulated phase in the distance $\ell$ is an integer multiple of $\pi$ [16-18]. We show below that the segmented wire exhibits resonant localization when the segment lengths are narrowly distributed around a given value $\ell_{0}$. This resonant localization occurs not only in the regime of one propagating mode, where one normally expects the fully $1 \mathrm{D}$ behavior, but also in regions with several propagating modes.

Localization in quasi-1D systems has been considered in wires with bulk and surface disorder (see, e.g., Ref. [19]

*1lorens.serra@uib.es and references therein). Indeed, analytical models based on Fokker-Planck equations have been developed for both types of disordered waveguides [20-22], as well as field-theoretic equivalent approaches [23,24]. A disorder-to-chaos transition when varying the degree of edge corrugation of a quasi-1D waveguide was recently predicted in Ref. [25]. Quasi-1D tight-binding and analytical models with correlated disorder were discussed in Refs. [26,27] and cylindrical shells were studied in Ref. [28]. Those studies, however, did not focus on the resonant behavior of the corresponding quasi-1D models. Our work is an approach to the resonant localization of the segmented wire, emphasizing the physical modeling of the 2D wire bends, complementing more methodological approaches focusing on the fundamentals of localization theory.

The paper is organized as follows. Section II presents the model and briefly describes the transport problem and the calculation of the vertex scattering matrix. Section III contains the definitions of the localization properties and Sec. IV contains the results. Finally, the conclusions of the work are summarized in Sec. V.

\section{SEGMENTED WIRE MODEL}

\section{A. Model definition}

We consider a quantum waveguide formed by joining straight 2D channels of width $d$. The joining vertices are described by simple circular bends of radius $R$ and angle $2 \theta$. Figure 1 sketches the system definitions. A set of values $\left\{\ell_{i}, \alpha_{i}, R_{i} ; i=1, \ldots, N_{v}\right\}$, with $N_{v}$ the number of vertices, gives a particular physical realization of the segmented wire. We are interested in the statistical properties when these values vary randomly within given intervals

$$
\begin{gathered}
\alpha_{i} \in\left[-\alpha_{0}, \alpha_{0}\right], \\
\ell_{i} \in\left[\ell_{0}\left(1-p_{\ell}\right), \ell_{0}\left(1+p_{\ell}\right)\right], \\
R_{i} \in\left[R_{0}\left(1-p_{R}\right), R_{0}\left(1+p_{R}\right)\right],
\end{gathered}
$$

where $\alpha_{0}, \ell_{0}, R_{0}, p_{\ell}$, and $p_{R}$ characterize the parameter ranges of random variation. The parameters $p_{\ell}$ and $p_{R}$ are dimensionless and fulfill $0 \leqslant p_{\ell, R} \leqslant 1$. They represent the maximum random variation (in relative terms) of the 
(a)

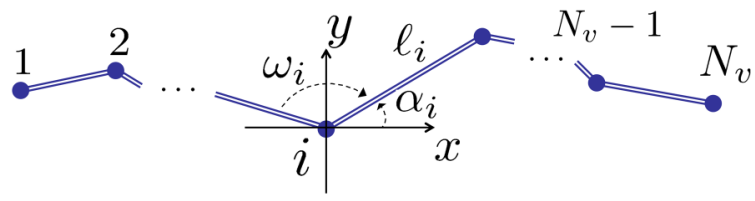

(b)

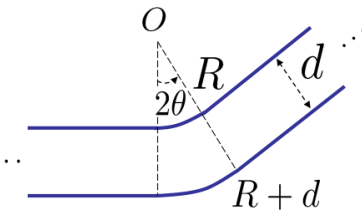

(c)

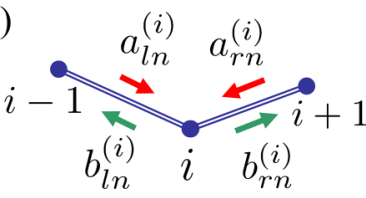

FIG. 1. (a) Sketch of a segmented 2D planar nanowire showing the definitions of the random angles $\alpha_{i}$ and $\omega_{i}$ for each vertex and the segment lengths $\ell_{i}$. (b) Model of the vertex as a circular bend, with the definitions of the bend radius $R$ and angle $2 \theta$. (c) Notation for the scattering amplitudes for left and right incidence on vertex $i$.

segment lengths around $\ell_{0}$ and of the vertex radius around $R_{0}$, respectively.

\section{B. Transport problem}

Each vertex is characterized by a scattering matrix relating input and output wave amplitudes

$$
\left(\begin{array}{l}
b_{l}^{(i)} \\
b_{r}^{(i)}
\end{array}\right)=\left(\begin{array}{ll}
r^{(i)} & t^{(i)} \\
t^{(i)} & r^{(i)}
\end{array}\right)\left(\begin{array}{l}
a_{l}^{(i)} \\
a_{r}^{(i)}
\end{array}\right)
$$

where the notation of Fig. 1(c) is used. For the case of multiple propagating modes, the input and output amplitudes in Eq. (4) correspond to vectors. For instance, $a_{l}^{(i)} \equiv\left(a_{l 1}^{(i)}, a_{l 2}^{(i)}, \ldots, a_{l N_{p}}^{(i)}\right)$, with $N_{p}$ the total number of propagating modes. Analogously, the transmission and reflection coefficients become matrices $t^{(i)} \equiv t_{n n^{\prime}}^{(i)}$ and $r^{(i)} \equiv r_{n n^{\prime}}^{(i)}$, respectively, with $n, n^{\prime}=1, \ldots, N_{p}$.

There is a relation between the input and output amplitudes for successive vertices. For instance, the right output from vertex $i-1$ in mode $n$ coincides with the left input for vertex $i$, with a phase, i.e., $b_{r n}^{(i-1)}=a_{l n}^{(i)} \exp \left(-i k_{n} l_{i-1}\right)$. A closed system of linear equations is obtained assuming unit left incidence on vertex 1 in mode $n_{i}$. The linear system of $4 N_{p} N_{v}$ equations reads

$$
\begin{aligned}
& a_{l n}^{(i)}=\delta_{n, n_{i}} \quad(i=1), \\
& a_{r n}^{(i)}=0 \quad\left(i=N_{v}\right), \\
& b_{l n}^{(i)}-a_{r n}^{(i-1)} \exp \left(-i k_{n} l_{i-1}\right)=0 \quad(i \neq 1), \\
& b_{r n}^{(i)}-a_{l n}^{(i+1)} \exp \left(-i k_{n} l_{i}\right)=0 \quad\left(i \neq N_{v}\right), \\
& b_{l n}^{(i)}-\sum_{n^{\prime}} r_{n n^{\prime}}^{(i)} a_{l n^{\prime}}^{(i)}-\sum_{n^{\prime}} t_{n n^{\prime}}^{(i)} a_{r n^{\prime}}^{(i)}=0 \quad\left(i=1, \ldots, N_{v}\right) \text {, } \\
& b_{r n}^{(i)}-\sum_{n^{\prime}} t_{n n^{\prime}}^{(i)} a_{l n^{\prime}}^{(i)}-\sum_{n^{\prime}} r_{n n^{\prime}}^{(i)} a_{r n^{\prime}}^{(i)}=0 \quad\left(i=1, \ldots, N_{v}\right),
\end{aligned}
$$

where $n$ and $n^{\prime}$ are mode indices ranging from 1 to $N_{p}$. The solution of the linear system yields the output amplitudes on each vertex as a function of the incidence mode $n_{i}, b_{r n}^{(i)}\left(n_{i}\right)$. The total transmission is obtained by adding the modulus squared of the right output amplitudes on the last vertex $N_{v}$ as

$$
T=\sum_{n_{i} n_{o}}\left|b_{r n_{o}}^{\left(N_{v}\right)}\left(n_{i}\right)\right|^{2} .
$$

Once the scattering matrices are known, the linear system can be efficiently solved in a numerical way for quite large values of the number of segments $N_{v}$. The computer solution is also fast enough to allow for an statistical analysis with the random variation of the parameters [Fig. 1(a)].

\section{Vertex scattering matrix}

The transmission and reflection matrices $t$ and $r$ for a single vertex are a required input of the model. We describe each vertex as a circular bend of the 2D wire [Fig. 1(b)], a problem that was studied in Refs. [2-5]. In particular, we follow the approach of Ref. [4], which relies on the separability of Schrödinger's equation in the bend region in radial and angular parts. Using polar coordinates for the bend region of Fig. 1(b), with $\rho$ the distance to $O$ and $\phi$ the azimuthal angle, the wave function may be factorized as $P_{|v|}(\rho) \exp ( \pm|v| \phi)$. The eigenvalue problem given in Eq. (2b) of Ref. [4] determines both $v^{2}$ and $P_{|v|}(\rho)$. For completeness, we repeat here the eigenvalue problem

$$
\rho^{2} \frac{d^{2} P_{|v|}(\rho)}{d \rho^{2}}+\rho \frac{d P_{|v|}(\rho)}{d \rho}+\frac{2 m E}{\hbar^{2}} \rho^{2} P_{|v|}(\rho)=-v^{2} P_{|v|}(\rho) .
$$

Equation (7) is not apparently Hermitian due to the nonsymmetric operators $\rho^{2} d^{2} / d \rho^{2}$ and $\rho d / d \rho$. However, the transformation $P_{|v|}=\sqrt{\rho} F_{|v|}$ leads to

$$
\rho \frac{d^{2}}{d \rho^{2}} \rho F_{|v|}(\rho)+\left(\frac{1}{4}+\frac{2 m E}{\hbar^{2}} \rho^{2}\right) F_{|\nu|}(\rho)=-v^{2} F_{|\nu|}(\rho),
$$

which, being symmetric, can be numerically diagonalized with a grid discretization and standard routines for symmetric matrices. As $v^{2}$ is thus a real value, $v$ can be either real or purely imaginary, which describe evanescent and propagating angular waves in the bend, respectively. Determining in this way the bend modes, the matching conditions at the interfaces [dashed lines of Fig. 1(b)] yield the required scattering matrices. As mentioned in Ref. [4], by truncating the number of bend modes and straight wire modes to the same value, a linear system of equations replaces the matching conditions. We have implemented that method and checked that our solution reproduces the transmission and reflection probabilities given in Ref. [4].

It was shown in Ref. [3] that at low energies the circular bend may be approximated by a 1D square well of depth $V_{0}=$ $\hbar^{2} / 2 m \bar{R}^{2}$ and width $2 a=2 \bar{R} \theta$, where $\bar{R}=\sqrt{R(R+d)}$ is the average effective radius. In this approximation the scattering matrices are of course analytical.

\section{LOCALIZATION PROPERTIES}

The localization length $\ell_{\text {loc }}$ is a characteristic distance such that random segmented wires whose total length $L$ fulfills $L \gg \ell_{\text {loc }}$ present a statistical distribution of $\ln (T)$ that is 
Gaussian (normal) distributed around a mean value. Of course, the localization length depends on the system parameters as well as on the energy $E$ of the transport electrons. Deeply in the localized regime $\left(L \gg \ell_{\text {loc }}\right)$ the transmission is in general greatly quenched for the huge majority of system realizations. It is therefore very relevant to characterize the parameter dependence of the localization length, as this is crucial for the electrical properties in coherent transport through the system.

In shorter wires $\left(L<\ell_{\mathrm{loc}}\right)$ transport is diffusive and typical of metallic conductors characterized by Ohm's law. In this case there is a linear relation between the electrical resistance and total length $L$. That is, in our model, we may expect a regime such that

$$
\left\langle\frac{1}{T}\right\rangle=\frac{1}{N_{p}}+\frac{1}{N_{p} \ell_{\Omega}}\langle L\rangle,
$$

where the averages are regarding system realizations and we have defined a diffusive (Ohmic) length $\ell_{\Omega}$ that characterizes the electron mean free path. The constant contribution $1 / N_{p}$ ( $N_{p}$ is the number of propagating modes) in Eq. (9) represents the contact resistance, present even without any scattering effect. The localization length and mean free path are actually related by $\ell_{\mathrm{loc}} \approx N_{p} \ell_{\Omega}$, a relation that we have explicitly checked for segmented wires (see also Ref. [29]). For completeness, besides the localized and diffusive regimes, the so-called ballistic regime corresponds to $L \ll \ell_{\Omega}$ such that only the contact resistance contribution matters in Eq. (9).

Yet another characteristic length may be obtained using a semiclassical approximation. In a semiclassical description the scatterers representing the vertices add up their effects incoherently, yielding a total transmission $T_{s c}$ that is independent of the segments length $\ell_{0}$,

$$
\frac{1}{T_{s c}}-\frac{1}{N_{p}}=\sum_{i=1}^{N_{v}}\left(\frac{1}{T^{(i)}}-\frac{1}{N_{p}}\right),
$$

where $T^{(i)} \equiv\left|t^{(i)}\right|^{2}$ is the transmission probability corresponding to vertex $i$. As the mean total length is $\langle L\rangle \approx N_{v} \ell_{0}$, Eq. (10) yields an Ohmic scaling similar to Eq. (9),

$$
\frac{1}{T_{s c}}=\frac{1}{N_{p}}+\frac{1}{\ell_{s c}}\langle L\rangle
$$

where we have defined a semiclassical length

$$
\ell_{s c}=\frac{\ell_{0}}{\left(\frac{1}{N_{v}} \sum_{i} \frac{1}{T^{(i)}}\right)-\frac{1}{N_{p}}} .
$$

As shown below, $\ell_{s c}$ yields an estimate of $\ell_{\text {loc }}$ that averages all possible oscillations and resonances due to quantum interference.

The Ohmic regime is sometimes referred to as diffusive or metallic and it is characterized by a relatively low transmission as compared to the maximum value allowed by the conductance quantization of the channel. Within the random matrix theory, this is a regime of universal conductance fluctuations [10], the transmission being normally distributed with a statistical dispersion $\Delta T=\sqrt{2 / 15}$ (in systems with time reversal symmetry like ours). On the other hand, in the
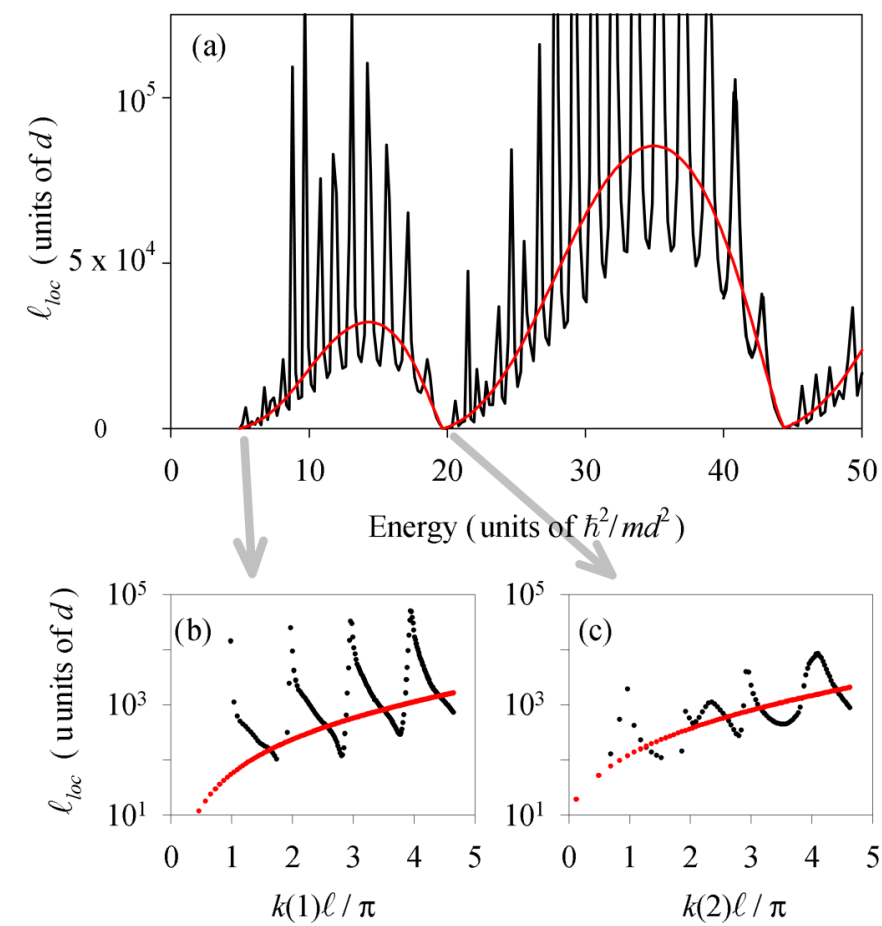

FIG. 2. Localization length as a function of energy. (b) and (c) show the dependence on mode wave number for the energy ranges around the first and second onsets for mode activation. The red (gray) line and symbols are the semiclassical results $\ell_{s c}$ defined in Eq. (12). The parameters are $\ell_{0}=10 d, R_{0}=0.2 d, p_{\ell}=0.01, p_{R}=0.01$, and $\alpha_{0}=60^{\circ}$.

ballistic limit the transmission reaches the quantized maximum values allowed by the number of propagating modes $N_{p}$. We expect this regime only in short-enough wires and relatively high energies, such that scattering effects become negligible.

\section{RESULTS}

Figure 2 shows the energy dependence of $\ell_{\text {loc }}$ in a segmented wire. A conspicuous resonant behavior can be seen, with closely lying spikes and an overall beating pattern corresponding to the successive activation of propagating modes. The beating is accurately reproduced by the semiclassical length $\ell_{s c}$ [red (gray) line], which nicely averages the resonant oscillations. The resonances occur when an integer number of wavelengths fit in the segment length $\ell_{0}$, i.e., $k(i) \ell_{0}=n \pi$, with $n$ an integer. This resonant condition does not depend on the vertex parameters $\alpha$ and $R_{0}$, or on $p_{R}$, but it quickly degrades when $p_{\ell}$ increases, as can be seen in Fig. 3. As shown in this figure, a dispersion of $\pm 10 \%$ is enough to greatly reduce the resonance peaks. The sensitivity of the peak heights to parameters other than $p_{\ell}$ is much smaller. The resonant behavior with one propagating mode is qualitatively similar to the behavior in strictly 1D systems discussed in Refs. [17,18]. We stress, however, that we also find similar resonances in higher-energy regions, where more modes become propagating.

The crossover between diffusive and localized regimes of disordered wires is known to be characterized by a nontrivial evolution of the $T$ and $\ln (T)$ distributions [25,30-32]. We 


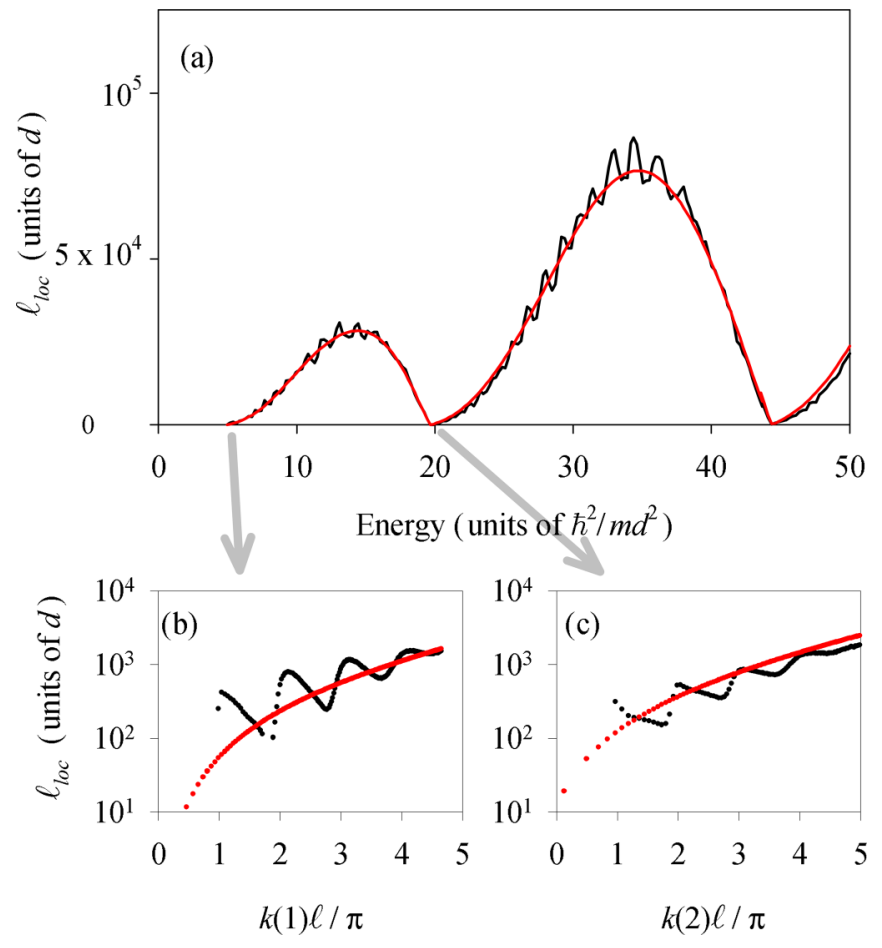

FIG. 3. Same as Fig. 2 but with $p_{\ell}=0.1$.

explore whether the crossover is greatly affected by the resonant condition or not. More specifically, we choose parameter sets corresponding to a maximum and a minimum in localization length and check the evolution with varying number of segments. Figure 4 shows that even when the localization length changes by more than an order of magnitude with a small energy change (spiking behavior in Fig. 2), the qualitative evolution of the probability distributions is very similar.

The results of Figs. 4(a) and 4(b) correspond to $L \approx 2 \ell_{\text {loc }}$, just entering the localized regime. They show a long-tail $T$ distribution with a change of behavior at $T=1$. On the other hand, $\ln (T)$ is given by an asymmetric Gaussian in this region. Figures 4(c) and 4(d) correspond to the middle of the crossover with $L \approx \ell_{\text {loc }}$ and show a rather flat distribution of transmissions with a dip at $T \approx 0[33,34]$. Figures 4(e) and 4(f) signal the beginning of the diffusive regime $L \approx 0.5 \ell_{\text {loc }}$ and show a kink at $T=1$ separating two regions in the $T$ distribution. These crossover features are already known and they agree well with the results of studies of disordered wires [30-32]. They are nevertheless shown here to stress the similar evolution for widely different localization lengths due to resonance. It is worth mentioning that when the localization length is very short, as for the red (gray) dots in Fig. 4, the transition from quasiballistic to localized is more abrupt, leaving a quite reduced diffusive range. We attribute to this enhanced quasiballistic behavior the increased kink at $T \approx 1$ of the red (gray) dots in Fig. 4(e), as compared with the black ones.

We discuss next the mean conductance of a wire with a fixed number of segments (Figs. 5 and 6). The conductance shows a general tendency to increase in discrete steps as the energy increases, which is typical of quantum wires. The conductance

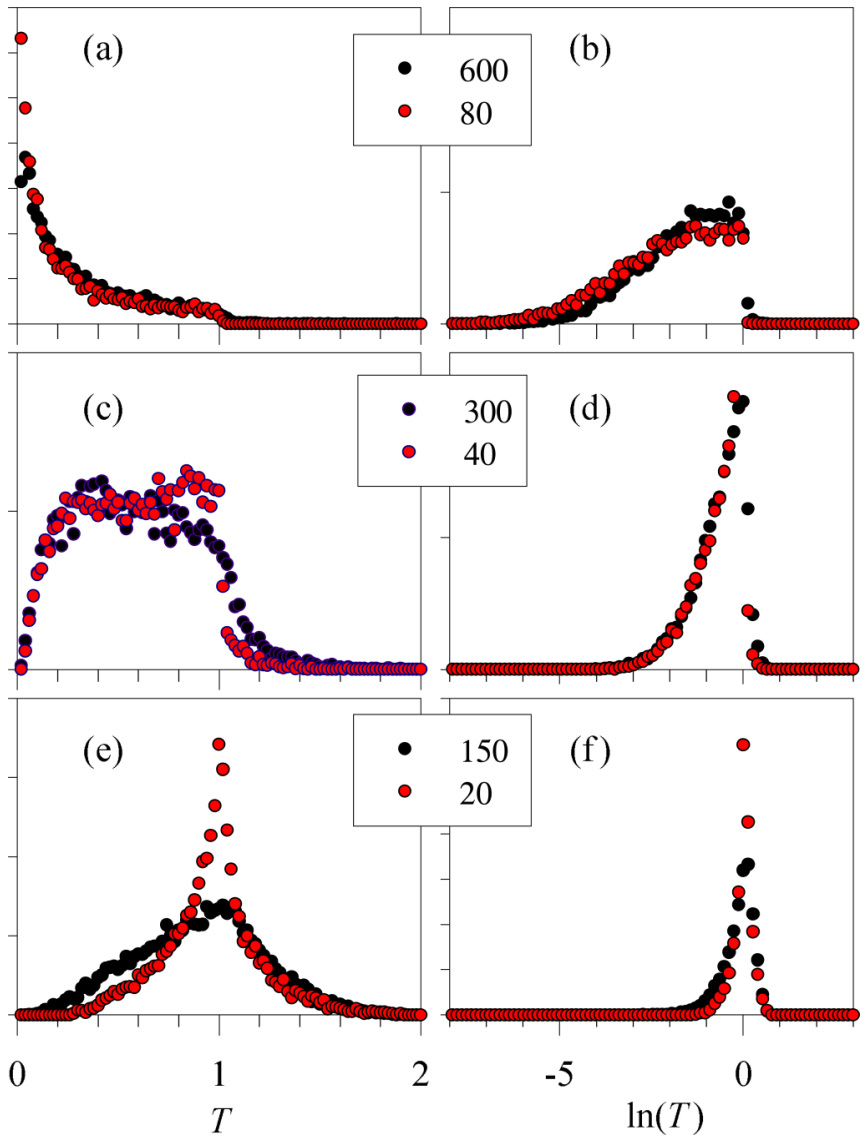

FIG. 4. Probability distribution of $T$ (left) and $\ln (T)$ (right) in the crossover regime. The number of segments corresponding to each symbol varies in each panel as indicated. Black circles correspond to an energy $E=19.8 \hbar^{2} / m d^{2}$ for which the localization length is $\ell_{\mathrm{loc}} \approx 3000 d$. On the other hand, red (gray) circles correspond to $E=20.1 \hbar^{2} / m d^{2}$ and $\ell_{\mathrm{loc}} \approx 280 d$. The rest of the parameters are the same as in Fig. 2.

plateaus, however, are distorted in remarkable ways. First we notice in Fig. 5 the already mentioned resonant oscillations, present at the beginning of each plateau for the smaller $N_{v}$ and eventually extending to all the plateau for the larger $N_{v}$ 's. A pronounced conductance dip is also observed at the beginning of each plateau. For the shorter wires the plateaus saturate at the quantized values, while in the longer ones there is no clear saturation and the transmission is in general much lower than the corresponding quantized values.

The physics implied by Fig. 5 can be understood as a typical evolution of a finite-wire conductance with increasing energy: from a localized regime near the plateau onset to an Ohmic (diffusive) regime and eventually reaching a ballistic regime if the wire is short enough. The localized regime occurs at the plateau onset, where the localization length is small (Figs. 2 and 3). As the energy increases, an Ohmic regime is reached, characterized by a sizable dispersion of the transmissions and by the linearity of the inverse transmission with length. In short wires [Fig. 5(a)] the system may also reach the quantum ballistic regime, with a quantized unitary transmission and vanishing dispersion. 

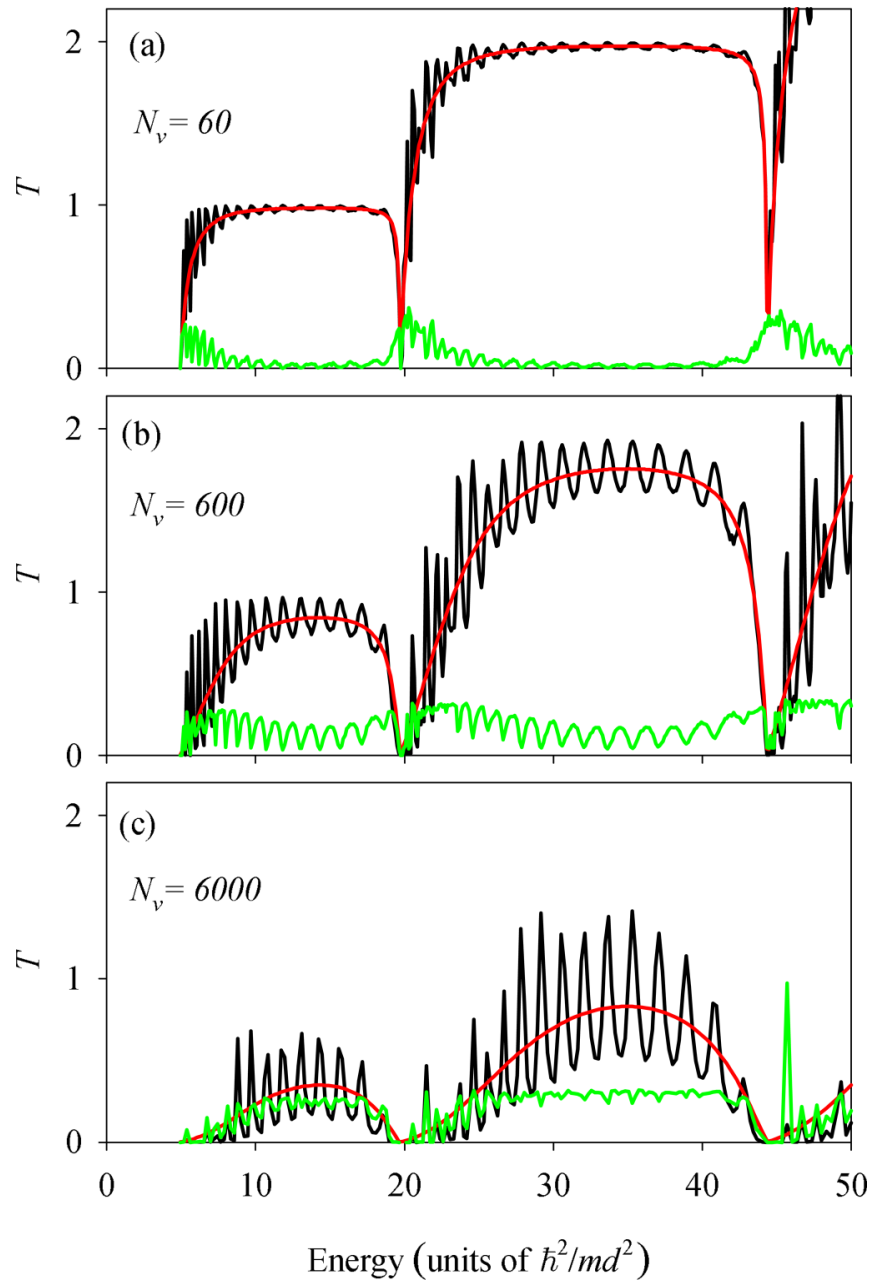

FIG. 5. Mean conductance as a function of energy for increasing number of segments as indicated in each panel. The same parameters as in Fig. 2 have been used. The green (light gray) line shows the dispersion of the statistical distribution of transmissions, while the red (gray) line is the semiclassical result $T_{s c}$.

As with the localization lengths, the strong transmission oscillations due to wave number quantization are quenched if the segment lengths vary by a sizable amount. Figure 6 shows the transmission with $p_{\ell}=0.1$. In this case, there is a better correspondence to the semiclassical result.

\section{CONCLUSION}

A model of random segmented 2D wire with circular bends has been presented. We focused on the scattering induced by the bends and how this leads to the emergence of localization. A strong resonant behavior is predicted when the segments are all of very similar lengths. A spiking behavior of the localization length is found, not only with a single propagating mode, but also in the presence of several modes. A beating pattern of the spiking is accurately reproduced by a semiclassical model, averaging quantum oscillations and resonances. The localization resonances are reduced when the distribution of segment lengths gets broader.

The localized-diffusive crossover is shown to agree with the known behaviors from disordered wires. The same qualitative
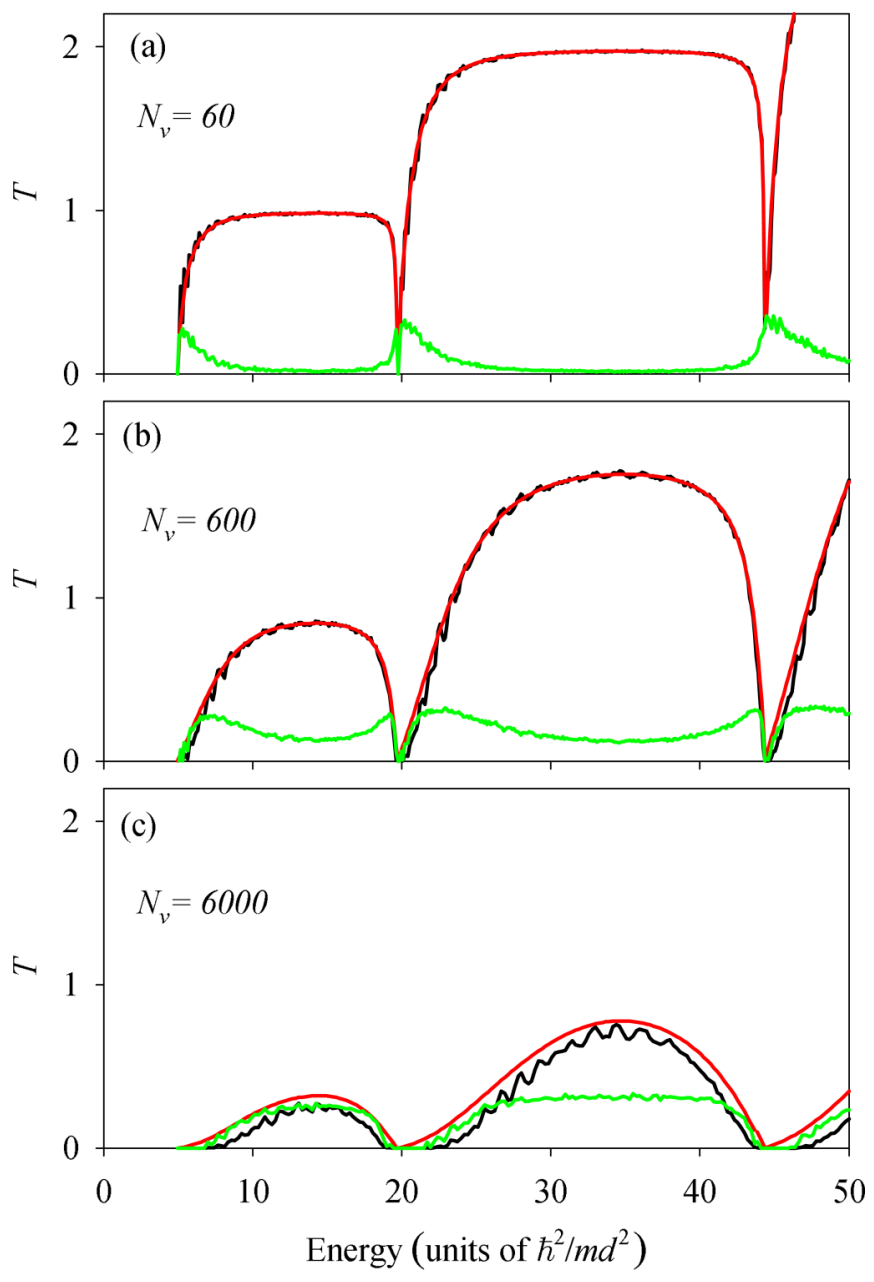

FIG. 6. Same as Fig. 5 but with $p_{\ell}=0.1$.

evolution of the $T$ and $\ln (T)$ distributions is found for large and small localization lengths, moving across the resonance spikes. For short localization lengths the diffusive regime is greatly reduced, yielding a more abrupt evolution from ballistic to localized cases. A fixed-length wire typically evolves with increasing energy from localization at the beginning of each transmission plateau to a diffusive regime and to ballistic behavior towards the plateau end. The ballistic regime may be reached only if the wire is short enough.

The localization properties discussed in this work are very likely also valid for $2 \mathrm{D}$ segmented wires with arbitrary bends (other than circular), as well as for bends in 3D tubes [1,35,36]. The computational burden is however significantly larger in these geometries, which makes the statistical analysis rather costly. Another interesting extension of the present work is studying spin-sensitive scattering on the bends, as introduced by spin-orbit coupling.

\section{ACKNOWLEDGMENTS}

This work was funded by MINECO (Spain) (Grant No. FIS2014-52564), CAIB (Spain) (Conselleria d'Educació, Cultura $\mathrm{i}$ Universitats), and FEDER. 
[1] J. T. Londergan, J. P. Carini, and D. P. Murdock, Binding and Scattering in Two-Dimensional Systems (Springer, Berlin, 1999).

[2] H. Wu, D. W. L. Sprung, and J. Martorell, Effective onedimensional square well for two-dimensional quantum wires, Phys. Rev. B 45, 11960 (1992).

[3] D. W. L. Sprung, H. Wu, and J. Martorell, Understanding quantum wires with circular bends, J. Appl. Phys. 71, 515 (1992).

[4] F. Sols and M. Macucci, Circular bends in electron waveguides, Phys. Rev. B 41, 11887 (1990).

[5] C. S. Lent, Transmission through a bend in an electron waveguide, Appl. Phys. Lett. 56, 2554 (1990).

[6] A. A. Sousa, A. Chaves, T. A. S. Pereira, G. A. Farias, and F. M. Peeters, Quantum tunneling between bent semiconductor nanowires, J. Appl. Phys. 118, 174301 (2015).

[7] C. Estarellas and L. Serra, A scattering model of 1d quantum wire regular polygons, Superlattices Microstruct. 83, 184 (2015).

[8] A. Sitek, L. Serra, V. Gudmundsson, and A. Manolescu, Electron localization and optical absorption of polygonal quantum rings, Phys. Rev. B 91, 235429 (2015).

[9] P. W. Anderson, Absence of diffusion in certain random lattices, Phys. Rev. 109, 1492 (1958).

[10] C. W. J. Beenakker, Random-matrix theory of quantum transport, Rev. Mod. Phys. 69, 731 (1997).

[11] B. Kramer and A. MacKinnon, Localization: Theory and experiment, Rep. Prog. Phys. 56, 1469 (1993).

[12] J. B. Pendry, Symmetry and transport of waves in onedimensional disordered systems, Adv. Phys. 43, 461 (1994).

[13] L. I. Deych, A. A. Lisyansky, and B. L. Altshuler, Singleparameter scaling in one-dimensional anderson localization: Exact analytical solution, Phys. Rev. B 64, 224202 (2001).

[14] S. Datta, Electronic Transport in Mesoscopic Systems (Cambridge University Press, Cambridge, 1997).

[15] P. A. Mello and K. Narendra, Quantum Transport in Mesoscopic Systems: Complexity and Statistical Fluctuations (Oxford University Press, Oxford, 2004).

[16] M. Diaz, P. A. Mello, M. Yepez, and S. Tomsovic, Wave transport in one-dimensional disordered systems with finitewidth potential steps, Europhys. Lett. 97, 54002 (2012).

[17] I. F. Herrera-González, F. M. Izrailev, and N. M. Makarov, Resonant enhancement of Anderson localization: Analytical approach, Phys. Rev. E 88, 052108 (2013).

[18] M. Díaz, P. A. Mello, M. Yépez, and S. Tomsovic, Wave transport in one-dimensional disordered systems with finite-size scatterers, Phys. Rev. B 91, 184203 (2015).

[19] L. S. Froufe-Pérez, M. Yépez, A. García-Martín, and J. J. Sáenz, in Condensed Matter Physics: IV Mexican Meeting on Experimental and Theoretical Physics: Symposium on Condensed Matter Physics, edited by M. Martinez-Mares and J. A. Moreno-Razo, AIP Conf. Proc. No. 1319 (AIP, New York, 2010), p. 29.
[20] O. N. Dorokhov, Transmission coefficient and the localization length of an electron in $N$ bound disordered systems, JETP Lett. 36, 318 (1982) [Pis'ma Zh. Eksp. Teor. Fiz. 36, 259 (1982)].

[21] P. A. Mello, P. Pereyra, and N. Kumar, Macroscopic approach to multichannel disordered conductors, Ann. Phys. (N.Y.) 181, 290 (1988).

[22] L. S. Froufe-Pérez, M. Yépez, P. A. Mello, and J. J. Sáenz, Statistical scattering of waves in disordered waveguides: From microscopic potentials to limiting macroscopic statistics, Phys. Rev. E 75, 031113 (2007).

[23] K. B. Efetov, Supersymmetry and theory of disordered metals, Adv. Phys. 32, 53 (2006).

[24] P. W. Brouwer and K. Frahm, Quantum transport in disordered wires: Equivalence of the one-dimensional $\sigma$ model and the Dorokhov-Mello-Pereyra-Kumar equation, Phys. Rev. B 53, 1490 (1996).

[25] A. Alcázar-López and J. A. Méndez-Bermúdez, Disorder-tochaos transition in the conductance distribution of corrugated waveguides, Phys. Rev. E 87, 032904 (2013).

[26] I. F. Herrera-González, J. A. Méndez-Bermúdez, and F. M. Izrailev, Transport through quasi-one-dimensional wires with correlated disorder, Phys. Rev. E 90, 042115 (2014).

[27] F. M. Izrailev, N. M. Makarov, and M. Rendón, Gradient and amplitude scattering in surface-corrugated waveguides, Phys. Rev. B 72, 041403 (2005).

[28] L. Serra and M.-S. Choi, Conductance of tubular nanowires with disorder, Eur. Phys. J. B 71, 97 (2009).

[29] A. García-Martín, J. A. Torres, J. J. Sáenz, and M. NietoVesperinas, Transition from diffusive to localized regimes in surface corrugated optical waveguides, Appl. Phys. Lett. 71, 1912 (1997).

[30] K. A. Muttalib and P. Wölfle, "One-sided" Log-Normal Distribution of Conductances for a Disordered Quantum Wire, Phys. Rev. Lett. 83, 3013 (1999).

[31] A. García-Martín and J. J. Sáenz, Universal Conductance Distributions in the Crossover Between Diffusive and Localization Regimes, Phys. Rev. Lett. 87, 116603 (2001).

[32] V. A. Gopar, K. A. Muttalib, and P. Wölfle, Conductance distribution in disordered quantum wires: Crossover between the metallic and insulating regimes, Phys. Rev. B 66, 174204 (2002).

[33] X. Wang, Q. Li, and C. M. Soukoulis, Scaling properties of conductance at integer quantum Hall plateau transitions, Phys. Rev. B 58, 3576 (1998).

[34] V. Plerou and Z. Wang, Conductances, conductance fluctuations, and level statistics on the surface of multilayer quantum hall states, Phys. Rev. B 58, 1967 (1998).

[35] C. Ortix and J. van den Brink, Effect of curvature on the electronic structure and bound-state formation in rolled-up nanotubes, Phys. Rev. B 81, 165419 (2010).

[36] G. Cuoghi, A. Bertoni, and A. Sacchetti, Effect of quasibound states on coherent electron transport in twisted nanowires, Phys. Rev. B 83, 245439 (2011). 